\title{
Reactions of $\mathrm{MoOCl}_{4}$ with 1-Methylimidazole, 1,4-Diaminobutane, 2-Methylpyridine, 4-Methylpiperidine, Trimethylsilylimidazole \& 1-Methylpyrrolidine
}

\author{
VIKAS MANGLA and GURSHARAN SINGH* \\ ${ }^{1}$ Research Scholar registered with Punjab Technical University, Kapurthala-144603, India. \\ ${ }^{2}$ Department of Applied Chemistry, Giani Zail Singh Campus College of Engineering \& Technology, \\ Dabwali Road, MRSPTU Bathinda-151001, India. \\ ${ }^{*}$ Corresponding author E-mail: gursharans82@gmail.com \\ http://dx.doi.org/10.13005/ojc/350324
}

(Received: May 11, 2019; Accepted: June 01, 2019)

\section{ABSTRACT}

\begin{abstract}
$\mathrm{MoOCl}_{4}$ reacts with 1-methylimidazole, 4-methylpiperidine and trimethylsilylimidazole (equimolar molar amounts) in solvent $\mathrm{CH}_{3} \mathrm{CN}$ to provide $\mathrm{MoO}_{2} \mathrm{Cl}_{4}\left(\mathrm{C}_{3} \mathrm{H}_{3} \mathrm{~N}_{2} \mathrm{CH}_{3}\right)$ [1], $\mathrm{MoOCl}_{4}\left(\mathrm{C}_{5} \mathrm{H}_{9} \mathrm{NHCH}_{3}\right)$ $\mathrm{CH}_{3} \mathrm{CN}[4]$ and $\mathrm{MoO}_{2} \mathrm{Cl}_{2}\left(\mathrm{C}_{3} \mathrm{H}_{4} \mathrm{~N}_{2}\right)$, [7]. $\mathrm{MoOCl}_{4}$ reacts with twice the moles of 1,4-diaminobutane, 2-methylpyridine, 4-methylpiperidine and 1-methylpyrrolidine in solvent $\mathrm{CH}_{3} \mathrm{CN}$ to provide: $\mathrm{MoOCl}_{4}\left(\mathrm{H}_{2} \mathrm{NC}_{4} \mathrm{H}_{8} \mathrm{NH}_{2}\right)_{2}$ [2], $\mathrm{MoOCl}_{4}\left(\mathrm{C}_{5} \mathrm{H}_{4} \mathrm{NCH}_{3}\right)$, [3], $\mathrm{MoOCl}_{4}\left(\mathrm{C}_{5} \mathrm{H}_{9} \mathrm{NHCH}_{3}\right)_{2},[5], \mathrm{MoOCl}_{4}\left(\mathrm{C}_{5} \mathrm{H}_{9} \mathrm{NHCH}_{3}\right)_{2}$, [6] and $\mathrm{MoO}_{2} \mathrm{Cl}_{4}\left(\mathrm{C}_{4} \mathrm{H}_{8} \mathrm{NCH}_{3}\right)_{2}$, [8]. Complexes have been studied by techniques: elemental quantitative analysis, FTIR, ${ }^{1} \mathrm{H}$ NMR, Mass (LC-MS).
\end{abstract}

Keywords: $\mathrm{MoOCl}_{4}$, 1-methylimidazole, 4-methylpiperidine, trimethylsilylimidazole, 1,4-diaminobutane, 1-methylpyridine, 1-methylpyrrolidine.

\section{INTRODUCTION}

Reactions of $\mathrm{MoOCl}_{4}$ with various ligands have been reported. Molybdenum in $\mathrm{MoOCl}_{4}$ being in VI oxidation state, it has the tendency to get reduced during reactions with ligands. Reactions may yield addition, substitution, reduction, rearrangement and polymerization products. Reactions of $\mathrm{MoOCl}_{4}$ in solvent $\mathrm{CH}_{2} \mathrm{Cl}_{2}$ have been reported ${ }^{1-5}$ by the author. Ligands are poorly soluble in solvent $\mathrm{CH}_{2} \mathrm{Cl}_{2}$ so reactions of $\mathrm{MoOCl}_{4}$ were also carried out and reported ${ }^{6-11}$ by the author in $\mathrm{CH}_{3} \mathrm{CN}$ medium.
Behavior of saturated $\mathrm{N}$-heterocyclic ligands (4-methylpiperidine, 1-methylpyrrolidine) and unsaturated $\mathrm{N}$-heterocyclic ligands (1-methylimidazole, trimethylsilylimidazole, 1-methylpyridine) towards $\mathrm{MoOCl}_{4}$ in solvent $\mathrm{CH}_{3} \mathrm{CN}$ at room temperature have been reported by the author in this paper.

FTIR, ${ }^{1}$ HNMR and Mass (LC-MS) spectra have helped in identifying the presence of the particular ligands in the compounds [1] to [8] synthesized. Further, Mass (LC-MS) spectra fragmentation pattern of these compounds supported their molecular formulae.

This is an Open Access article licensed under a Creative Commons license: Attribution 4.0 International (CC- BY). Published by Oriental Scientific Publishing Company @ 2018

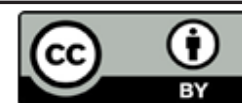




\section{Aim of Investigation}

Over the years, there has been increasing applications of Schiff bases and their transition metal complexes in biology, including antifungal, antiinflammatory, antibacterial, anticancer, antimalarial, antiviral activity. Such studies on complexes of Schiff bases 1-methylimidazole, trimethylsilylimidazole; 1-methylpyridine with transition metals have been scarcely reported. Efforts have been done to prepare such compounds of molybdenum.

Alkylation increases the basic character of piperidine and pyrrolidine. Reactions of simple piperidine and pyrrolidine with $\mathrm{MoOCl}_{4}$ have already been reported ${ }^{1,2}$ by the author. Increased basicity of alkylated piperidine and pyrrolidine is expected to impact the substitution/ addition and redox reactions with $\mathrm{MoOCl}_{4}$.

In view of the above, it was decided to carry out the current investigation.

\section{MATERIALS AND METHODS}

Precursor $\mathrm{MoOCl}_{4}$ was synthesized in the lab. by refluxing $\mathrm{SOCl}_{2}$ with $\mathrm{MoO}_{3}(\mathrm{CDH}, \mathrm{AR}$ Grade) for 5 hours. After the reaction, unreacted $\mathrm{SOCl}_{2}$ was removed with the help of vacuum and collected in in liquid nitrogen traps. Residue thus obtained was dark green in colour. It was treated with dry solvent $\mathrm{CH}_{2} \mathrm{Cl}_{2}$. Deep red solution was obtained. Red solution was passed through filtration unit having G-4 sintered glass crucible. $\mathrm{MoOCl}_{4}$ dark green crystals were obtained on evaporation of the filtrate.

Sigma-Aldrich ligands were used: 1-Methylimidazole (m.p./b.p. $-6^{\circ} \mathrm{C} / 124^{\circ} \mathrm{C}$ ), 4-Methylpiperidine (m.p./b.p. $4^{\circ} \mathrm{C} / 198^{\circ} \mathrm{C}$ ), Trimethylsilylimidazole (m.p./b.p. $-42^{\circ} \mathrm{C} / 93^{\circ}-94^{\circ} \mathrm{C}$ ), 1,4-Diaminobutane (m.p./b.p. $\left.25^{\circ}-28^{\circ} \mathrm{C} / 158^{\circ}-160^{\circ} \mathrm{C}\right)$, 2-Methylpyridine (m.p./b.p. $-70^{\circ} \mathrm{C} / 128^{\circ}-129^{\circ} \mathrm{C}$ ), 1-Methylpyrrolidine (m.p./ b.p. $-90^{\circ} \mathrm{C} / 76^{\circ}-80^{\circ} \mathrm{C}$ ). These ligands were vacuum dried. LR grade thionyl chloride (b.p. $76^{\circ}-78^{\circ} \mathrm{C}, \mathrm{CDH}$ ) was treated with quinoline for 2 days ( $250 \mathrm{~g} \mathrm{SOCl}_{2}$ to $50 \mathrm{~g}$ quinoline) to eliminate acidic impurities. Fractional distillation was carried out to obtain colourless thionyl chloride. Solvent $\mathrm{CH}_{3} \mathrm{CN}$ was dried by standard procedure.

Molybdenum and $\mathrm{Cl}^{-}$were determined by standard procedures ${ }^{12}$. C, H, N, O were determined with Thermo Finnigan Elemental Analyzer, ${ }^{1} \mathrm{H}-\mathrm{NMR}$ spectra have been recorded on Brucker Avance-II 400 (Fallanden) NMR in DMSO-d ${ }_{6}$, FTIR spectra in the range $4000-400 \mathrm{~cm}^{-1}$ were taken on PerkinElmer 400 FTIR Spectrometer (Germany), in $\mathrm{KBr}$ disks, LC-MS spectra were obtained in the range $0-1100 \mathrm{~m} / \mathrm{z}$ using WATERS, Q-TOF Micromass LC-MS (UK), at Panjab University, Chandigarh (India), SAIF/CIL facility.

\section{Preparation of compounds [1] - [8]}

One $100 \mathrm{ml} \mathrm{R.B.} \mathrm{flask} \mathrm{was} \mathrm{connected} \mathrm{to}$ a pressure dropping funnel fitted having teflon stop-cock. A magnetic bead was added in the flask. The whole unit was dried under vacuum flame drying. After cooling the unit, dry nitrogen gas was flushed into it. A known weight of $\mathrm{MoOCl}_{4}$ dissolved in dry $\mathrm{CH}_{3} \mathrm{CN}$ was taken in round bottomed flask. Equimolar or 1:2 molar amount of 1-methylimidazole, 4-methylpiperidine trimethylsilylimidazole, 1,4diaminobutane, 2-methylpyridine, 4-methylpiperidine or 1-methylpyrrolidine were dissolved in solvent $\mathrm{CH}_{3} \mathrm{CN}$ and taken in dropping funnel. The ligand from the dropping funnel was mixed with $\mathrm{MoOCl}_{4}$ in $\mathrm{rb}$ flask at room temperature with constant stirring. Products were filtered through G-4 bed of a filtration unit, under reduced pressure. Compounds prepared are very much sensitive to air and moisture. They tend to turn blue in colour. All reactions were carried out under oxygen free dry nitrogen gas atmosphere in vacuum line. Liquid nitrogen cooled traps were used to get rid of moisture, oxygen.

There seems to be disproportionation/ rearrangement during reactions. Filtrate or residue mentioned below the products, refers to the source from where the product has been isolated.

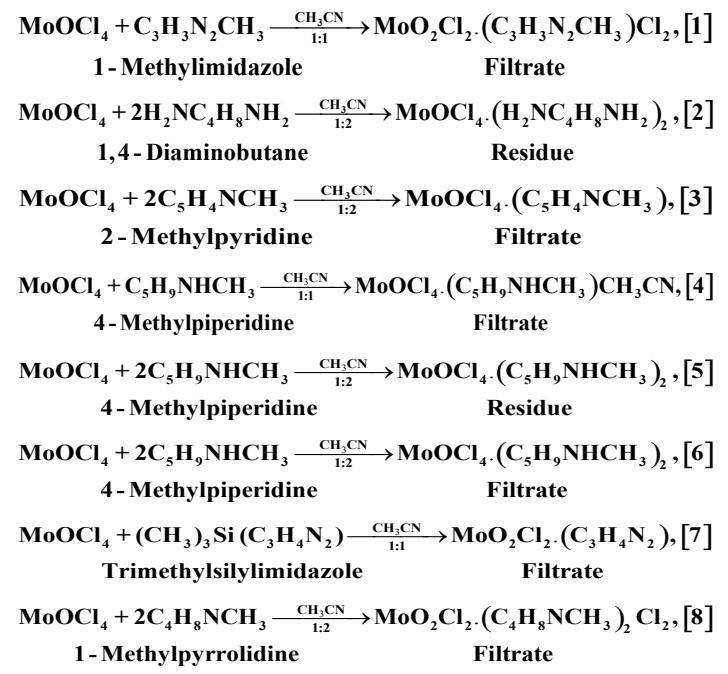




\section{RESULTS AND DISCUSSIONS}

\section{Analytical Measurements}

Compounds are very much sensitive to moisture and air. They are insoluble in less polar solvents like n-hexane, $\mathrm{CH}_{2} \mathrm{Cl}_{2}, \mathrm{CHCl}_{3}$, but are soluble in solvents like $\mathrm{CH}_{3} \mathrm{CN}$, DMSO and DMF of high polarity. These compounds have been formulated on basis of their elemental analysis and LC-MS studies (Table 1).

Table 1: (Elemental Analysis)

\begin{tabular}{|c|c|c|c|c|c|c|}
\hline \multirow{2}{*}{$\begin{array}{l}\text { Compounds } \\
\text { (Color/Formula Mass) }\end{array}$} & \multicolumn{5}{|c|}{$\%$ Observed (Theoretical) } & \multirow[b]{2}{*}{0} \\
\hline & Mo & $\mathrm{Cl}$ & C & $\mathrm{H}$ & $\mathrm{N}$ & \\
\hline \multirow{2}{*}{$\begin{array}{c}\mathrm{MoO}_{2} \mathrm{Cl}_{2} \cdot\left(\mathrm{C}_{3} \mathrm{H}_{3} \mathrm{~N}_{2} \mathrm{CH}_{3}\right) \mathrm{Cl}_{2},[1] \\
(\text { Green/352.0) }\end{array}$} & 27.72 & 40.92 & 14.12 & 2.27 & 8.11 & 8.72 \\
\hline & -27.27 & -40.34 & -13.63 & -1.7 & -7.95 & -9.09 \\
\hline \multirow{2}{*}{$\begin{array}{l}\mathrm{MoOCl}_{4} \cdot\left(\mathrm{H}_{2} \mathrm{NC}_{4} \mathrm{H}_{8} \mathrm{NH}_{2}\right)_{2}, \text { [2] } \\
\quad \text { (Light blue/434.0) }\end{array}$} & 21.23 & 32.23 & 21.19 & 6.28 & 12.34 & 3.82 \\
\hline & -22.12 & -32.72 & -22.12 & -6.45 & -12.9 & -3.68 \\
\hline \multirow{2}{*}{$\begin{array}{c}\mathrm{MoOCl}_{4} \cdot\left(\mathrm{C}_{5} \mathrm{H}_{4} \mathrm{NCH}_{3}\right),[3] \\
(\text { Black/347.0) }\end{array}$} & 26.95 & 40.45 & 20.23 & 2.17 & 4.74 & 4.57 \\
\hline & -27.66 & -40.92 & -20.74 & -2.02 & -4.03 & -4.61 \\
\hline \multirow{2}{*}{$\begin{array}{l}\mathrm{MoOCl}_{4} \cdot\left(\mathrm{C}_{5} \mathrm{H}_{9} \mathrm{NHCH}_{3}\right) \mathrm{CH}_{3} \mathrm{CN},[4] \\
\text { (Greyish blue/394.0) }\end{array}$} & 25.63 & 36.83 & 21.92 & 4.27 & 6.56 & 4.17 \\
\hline & -24.36 & -36.04 & -21.32 & -4.06 & -7.11 & -4.06 \\
\hline \multirow{2}{*}{$\begin{array}{c}\mathrm{MoOCl}_{4} \cdot\left(\mathrm{C}_{5} \mathrm{H}_{9} \mathrm{NHCH}_{3}\right)_{2},[5] \\
(\text { Dark brown/452.0) }\end{array}$} & 20.6 & 32.1 & 32.8 & 6.28 & 6.34 & 3.15 \\
\hline & -21.23 & -31.42 & -31.86 & -5.75 & -6.19 & -3.54 \\
\hline \multirow{2}{*}{$\begin{array}{l}\mathrm{MoOCl}_{4} \cdot\left(\mathrm{C}_{5} \mathrm{H}_{9} \mathrm{NHCH}_{3}\right)_{2},[6] \\
\text { (Greenish blue/452.0) }\end{array}$} & 21.7 & 30.9 & 17.81 & 5.74 & 5.85 & 3.13 \\
\hline & -21.23 & -31.42 & -31.86 & -5.75 & -6.19 & -3.54 \\
\hline \multirow{2}{*}{$\begin{array}{r}\mathrm{MoO}_{2} \mathrm{Cl}_{2} \cdot\left(\mathrm{C}_{3} \mathrm{H}_{4} \mathrm{~N}_{2}\right),[7] \\
\text { (Parrot green/267.0) }\end{array}$} & 36.67 & 27.43 & 14.36 & 2.14 & 10.57 & 11.23 \\
\hline & -35.95 & -26.59 & -13.48 & -1.5 & -10.48 & -11.98 \\
\hline \multirow{2}{*}{$\begin{array}{l}\mathrm{MoO}_{2} \mathrm{Cl}_{2} \cdot\left(\mathrm{C}_{4} \mathrm{H}_{8} \mathrm{NCH}_{3}\right)_{2} \mathrm{Cl}_{2},[8] \\
\quad \text { (Blue/440.0) }\end{array}$} & 22.33 & 33.1 & 27.72 & 5.15 & 5.95 & 6.93 \\
\hline & -21.82 & -33.27 & -27.27 & -5 & -6.36 & -7.27 \\
\hline
\end{tabular}

\section{FTIR Spectra}

Close proximity of vibrational frequencies of 1-methylimidazole ${ }^{13,14}$ with that of [1] shows the presence of this ligand in [1]. Nitrogen at position 3 of 1-methylimidazole makes a coordinate bond with molybdenum. On Mo-N coordination, there is increase in ring $\mathrm{C}=\mathrm{C}$ ring str. recorded at 1584.5 $\mathrm{cm}^{-1}, 1548.9 \mathrm{~cm}^{-1}$. There is also increase in ring $\mathrm{N}-\mathrm{C}$ str. observed at $1442.7 \mathrm{~cm}^{-1}$. This increase in frequencies is because of following 2 reasons:

(i) Inductive effect due to coordination with positive metal ion.

(ii) $\mathrm{d} \pi-\mathrm{p} \pi$ interactions dissipate the accumulation of negative charge.

This leads to increase in electron density in the ligand ring system. The greater the increase in ring frequency the stronger is the Mo-N coordinate bond. Presence of cis- $\mathrm{MoO}_{2}{ }^{2+}$ core ${ }^{15}$ in [1] is indicated by the presence of strong bands at $983.7 \mathrm{~cm}^{-1}$ and $918.1 \mathrm{~cm}^{-1}$ (Table 2).

$\mathrm{N}-\mathrm{H}$ stretching frequencies have been observed at $3413.1 \mathrm{~cm}^{-1}, 3080.0 \mathrm{~cm}^{-1}$ and 3012.5 $\mathrm{cm}^{-1}$ in [2] (Table 3). A strong $\mathrm{Mo}=\mathrm{O}$ stretching ${ }^{16},{ }^{17}$ at $920.0 \mathrm{~cm}^{-1}$ shows the presence of terminal
$\mathrm{Mo}=\mathrm{O}$. Bending mode due to $\mathrm{NH}_{2}$ observed in 1,4-diaminobutane ${ }^{18}$ at $1145 \mathrm{~cm}^{-1}$ is lowered to $1116.2 \mathrm{~cm}^{-1}$, because of Mo-N coordination.

Ring C-H stretching in 2-methylpyridine ${ }^{19-22}$ are obtained at $3137 \mathrm{~cm}^{-1}$ and $3066 \mathrm{~cm}^{-1}$. Ring $\mathrm{C}-\mathrm{H}$ stretching in [3] are observed at higher frequencies $3296.0 \mathrm{~cm}^{-1}$ and $3081.0 \mathrm{~cm}^{-1}$. C=N Str. in [3] was observed at higher frequency at $1625 \mathrm{~cm}^{-1}$. C-N stretching in [3] was observed at lower frequency at $1288.3 \mathrm{~cm}^{-1}$ (Table 4). All these observations indicate the presence of 2-methylpyridine in [3]. A strong $\mathrm{Mo}=\mathrm{O}$ stretching at $980.0 \mathrm{~cm}^{-1}$ in [3] shows the presence of terminal $\mathrm{Mo}=\mathrm{O}^{16,17}$.

There is $\mathrm{N}-\mathrm{H}$ stretching at $3283 \mathrm{~cm}^{-1}$ in 4-methylpiperidine ${ }^{23-26}$. Stretching at $3152.7 \mathrm{~cm}^{-1}$ in [4], $3088.7 \mathrm{~cm}^{-1}$ in [5] and $3088.7 \mathrm{~cm}^{-1}$ in [6] suggest presence of $\mathrm{N}-\mathrm{H}$ group in these compounds. Decrease in frequency is due to coordination of $\mathrm{N}-\mathrm{H}$ group through nitrogen atom in these compounds. Stretching at $980.8 \mathrm{~cm}^{-1}, 975.8 \mathrm{~cm}^{-1}$ and $979.2 \mathrm{~cm}^{-1}$ in [4], [5] and [6], respectively, refer to $\mathrm{Mo}=\mathrm{O}^{16,17}$ group in terminal position (Table 5).

$\mathrm{N}-\mathrm{H}$ stretching in imidazole ${ }^{27-29}$ are observed at $3724-3237 \mathrm{~cm}^{-1}$. [7] shows $\mathrm{N}-\mathrm{H}$ broad 
stretching at $3246.0 \mathrm{~cm}^{-1}$ due to hydrogen bonding in the solid state ( $\mathrm{KBr}$ disk). Close proximity of vibrational frequencies of imidazole ${ }^{27-29}$ with that of [7] shows the presence of this ligand in [7]. Nitrogen at position 3 of imidazole makes a coordinate bond with molybdenum. On Mo-N coordination, there is increase in ring $\mathrm{C}=\mathrm{C}$ ring str. recorded at 1615.0 $\mathrm{cm}^{-1}$ and $1584.0 \mathrm{~cm}^{-1}$. There is also increase in ring $\mathrm{N}-\mathrm{C}$ str. observed at $1436.6 \mathrm{~cm}^{-1}$. This increase in frequencies is because of the reasons already explained for [1]. Two bands attributable to the presence of stretching due to cis- $\mathrm{MoO}_{2}{ }^{2+}$ core ${ }^{15}$ are observed at $972.9 \mathrm{~cm}^{-1}$ and $916.4 \mathrm{~cm}^{-1}$ in [7] (Table 6).

Table 2: (FTIR frequencies in $\mathrm{cm}^{-1}$ )

\begin{tabular}{|c|c|c|}
\hline Mode & $\begin{array}{c}\mathrm{C}_{3} \mathrm{H}_{3} \mathrm{~N}_{2} \mathrm{CH}_{3} \\
\text { (1-Methylimidazole) }^{13,14}\end{array}$ & [1] \\
\hline Ring C-H str. & $3015 \mathrm{~m}, 2953 \mathrm{w}$ & $3291.8 \mathrm{vs}, 3151.0 \mathrm{~s}$ \\
\hline Ring $C=C$ str. & 1517 vs & $1584.5 \mathrm{~m}, 1548.9 \mathrm{w}$ \\
\hline Ring N-C str. & $1407 \mathrm{~m}$ & $1442.7 \mathrm{~m}$ \\
\hline $\mathrm{C}-\mathrm{H}$ in plane bending & $1106 \mathrm{~m}, 1085 \mathrm{~m}, 1033 \mathrm{vw}$ & $1155.7 \mathrm{w}, 1084.9 \mathrm{w}$ \\
\hline $\mathrm{C}-\mathrm{H}$ wagging, Ring twisting & $813 \mathrm{~s}, 772 \mathrm{~s}$ & $752.4 \mathrm{~s}$ \\
\hline Ring twisting & $638 \mathrm{~s}$ & 622.5 \\
\hline $\mathrm{N}-\mathrm{H}$ wagging, Ring twisting & 523 & $571.5,504.9 \mathrm{w}$ \\
\hline$v(\mathrm{Mo}=\mathrm{O})$ of cis- $\mathrm{MoO}_{2}{ }^{2+}$ core ${ }^{15}$ & --- & 983.7 vs, $918.1 \mathrm{w}$ \\
\hline
\end{tabular}

Table 3: (FTIR frequencies in $\mathrm{cm}^{-1}$ )

\begin{tabular}{ccc}
\hline Mode & $\mathrm{H}_{2} \mathrm{NC}_{4} \mathrm{H}_{8} \mathrm{NH}_{2}$ & [2] \\
& $(1,4$-Diaminobutane) & \\
\hline $\mathrm{N}-\mathrm{H}$ Str. & 3346,3280 & $3413.1 \mathrm{~s}, 3080.0 \mathrm{vs}, 3012.5 \mathrm{vs}$ \\
$\mathrm{CH}_{2}$ Str. & $2960-2875$ & $2946.5 \mathrm{sh}, 2881.5 \mathrm{sh}$ \\
$\mathrm{NH}_{2}$ Bending & 1606 & $1612.7 \mathrm{~m}$ \\
$\mathrm{CH}_{2}$ Deformation (strong) & $1497,1390,1353,1309$ & $1470.8 \mathrm{~m}, 1446.5 \mathrm{~s}$ \\
$\mathrm{NH}_{2}$ Bending & 1145 & $1283.2 \mathrm{~s}, 1116.2 \mathrm{~s}$ \\
$\mathrm{C}-\mathrm{N}$ sym str. (weak) & 1070 & $1028.3 \mathrm{~m}$ \\
$\mathrm{CH} \mathrm{H}_{2}$ Deformation (medium) & 863,738 & $872.8 \mathrm{~m}, 765.0 \mathrm{w}$ \\
Mo-N (Strong) & ---- & $499.7 \mathrm{~m}$ \\
Terminal v(Mo=O)16, 17 & --- & $920.0 \mathrm{~m}$ \\
\hline
\end{tabular}

Table 4:(FTIR frequencies in $\mathrm{cm}^{-1}$ )

\begin{tabular}{lcc}
\hline \multicolumn{1}{c}{ Mode } & $\begin{array}{c}\mathrm{C}_{5} \mathrm{H}_{4} \mathrm{NCH}_{3} \\
\text { (2-Methylpyridine) })^{1922}\end{array}$ \\
\hline Ring C-H Str. & $3137 \mathrm{~m}, 3086 \mathrm{~m}, 3066 \mathrm{~m}, 3012 \mathrm{~s}$ & $3296.0 \mathrm{~s}, 3081.0 \mathrm{~s}$ \\
Methyl C-H Str. & $2958 \mathrm{~m}$ & $2928.0 \mathrm{~s}, 2837.0 \mathrm{~s}$ \\
C=N Str. & $1596 \mathrm{vs}$ & $1625.0 \mathrm{~s}$ \\
Ring C-C Str. & $1589 \mathrm{~m}$ & $1617.0 \mathrm{~s}$ \\
Ring C-H in plane bending & $1477 \mathrm{~s}$ & $1538.1 \mathrm{~s}$ \\
Methyl C-H Asym. bending & $1461 \mathrm{~s}$ & $1469.1 \mathrm{~s}$ \\
Methyl C-H Sym. bending & $1377 \mathrm{w}$ & $1396.2 \mathrm{~m}$ \\
C-N Str. & $1295 \mathrm{~s}$, & $1288.3 \mathrm{~m}$ \\
C-CH3 Str. & $1237 \mathrm{~m}$ & $1234.5 \mathrm{w}$ \\
Ring C-H in plane bending & $1148 \mathrm{~m}$, & $1165.2 \mathrm{~s}$ \\
Ring C-C Str. & $1101 \mathrm{w}$ & $1108.5 \mathrm{w}, 1096.6 \mathrm{w}$ \\
Ring breathing & $1060 \mathrm{~s}$ & $1046.4 \mathrm{~m}$ \\
Ring C-H, C-C, C-N out of plane bending & $752 \mathrm{vs}$ & $770.0 \mathrm{~s}$ \\
Ring C-H out of plane bending & $731 \mathrm{~m}$ & - \\
Ring C-C out of plane bending & $629 \mathrm{~m}$ & $627.1 \mathrm{w}$ \\
Ring C-C-C in plane bending & $547 \mathrm{w}$ & 566.3 \\
C-CH3 Bending & 471 & $471.3 \mathrm{~s}$ \\
Terminal Mo=O16, 17 Str. & ---- & $980.0 \mathrm{~s}$ \\
\hline
\end{tabular}


Table 5:(FTIR frequencies in $\mathrm{cm}^{-1}$ )

\begin{tabular}{|c|c|c|c|c|}
\hline Mode & $\begin{array}{c}\mathrm{C}_{5} \mathrm{H}_{9} \mathrm{NHCH}_{3} \\
\text { (4-Methylpiperidine) }{ }^{23-26}\end{array}$ & [4] & {$[5]$} & {$[6]$} \\
\hline N-H Str. & $3283 \mathrm{~m}$ & $\begin{array}{l}3367.2 \mathrm{~s} \\
3152.7 \mathrm{~s}\end{array}$ & $\begin{array}{c}3377.1 \mathrm{sh} \\
3088.7 \mathrm{~s}\end{array}$ & $\begin{array}{c}3367.4 \mathrm{sh}, \\
3150.0 \mathrm{~s}\end{array}$ \\
\hline $\mathrm{CH}_{3}$ Sym. Str. & 2967 s, 2915 s & $2957.7 \mathrm{~s}$ & $2953.0 \mathrm{~s}$ & $2956.1 \mathrm{~s}$ \\
\hline Ring C-H Asym. Str. & 2800 s, 2732 m & 2808.1 w & $\begin{array}{l}2847.4 \mathrm{~s} \\
2791.5 \mathrm{~s}\end{array}$ & $2806.6 \mathrm{sh}$ \\
\hline Ring C-H Deformation & $\begin{array}{c}1456 \mathrm{~m}, 1448 \mathrm{~m} \\
1407.3 \mathrm{w}\end{array}$ & $\begin{array}{c}1610.7 \mathrm{~s} \\
1452.7 \mathrm{~m} \\
1451.8 \mathrm{~s}\end{array}$ & $\begin{array}{c}1609.0 \mathrm{sh} \\
1570.5 \mathrm{~s}\end{array}$ & $\begin{array}{l}1569.3 \mathrm{~s} \\
1452.1 \mathrm{~s}\end{array}$ \\
\hline Ring C-C Str. & 1386 w, $1323 \mathrm{~m}$ & $\begin{array}{l}1386.8 w \\
1301.3 w\end{array}$ & $1302.0 \mathrm{w}$ & $1303.9 \mathrm{w}$ \\
\hline C-N Str. & 1265 w, $1153 \mathrm{~m}$ & $1223.9 \mathrm{w}$ & $\begin{array}{l}1224.7 \mathrm{w} \\
1178.9 \mathrm{w}\end{array}$ & 1223.5 w \\
\hline Ring $\mathrm{C}-\mathrm{H}$ Bending & 1007 w, 983 w, 972 w & $\begin{array}{c}1065.9 w \\
1038.0 w \\
917.8 w\end{array}$ & $\begin{array}{c}1070.6 \mathrm{w} \\
1038.5 \mathrm{w} \\
954.9 \mathrm{~m}\end{array}$ & $\begin{array}{l}1068.9 \mathrm{w} \\
1038.1 \mathrm{w}\end{array}$ \\
\hline CH2 Rocking & $795 \mathrm{~m}, 771 \mathrm{~s}$ & $762.4 \mathrm{~s}$ & $\begin{array}{l}871.6 \mathrm{w} \\
722.1 \mathrm{~s}\end{array}$ & $\begin{array}{l}876.1 \mathrm{w}, \\
839.1 \mathrm{w}, \\
786.5 \mathrm{w}, \\
724.1 \mathrm{~m}\end{array}$ \\
\hline CNC Deformation & $571 \mathrm{~m}$ & $\begin{array}{l}568.2 \mathrm{~m}, \\
444.9 \mathrm{w}\end{array}$ & $\begin{array}{l}569.8 \mathrm{w}, \\
514.7 \mathrm{~m}, \\
447.9 \mathrm{w} \\
410.9 \mathrm{w}\end{array}$ & $\begin{array}{l}567.8 \mathrm{w} \\
496.3 \mathrm{w} \\
445.0 \mathrm{w} \\
407.9 \mathrm{w}\end{array}$ \\
\hline Terminal $v(\mathrm{Mo}=\mathrm{O})^{16,17}$ & -- & $980.8 \mathrm{~s}$ & $975.8 \mathrm{~s}$ & $979.2 \mathrm{~s}$ \\
\hline
\end{tabular}

Table 6: (FTIR frequencies in $\mathrm{cm}^{-1}$ )

\begin{tabular}{|c|c|c|}
\hline Mode & $\mathrm{C}_{3} \mathrm{H}_{4} \mathrm{~N}_{2}$ (Imidazole $)^{27-29}$ & {$[7]$} \\
\hline$v(\mathrm{~N}-\mathrm{H})$ & 3724 b, 3656 b, 3270, 3241, 3237 & $3246.0 \mathrm{~b}$ \\
\hline$v(\mathrm{C}-\mathrm{H})$ & 3196,3165 & $3149.1 \mathrm{~s}, 2993.1 \mathrm{sh}$ \\
\hline Ring $\cup(C=C)$ & 1558,1500 & $1615.0 \mathrm{~s}, 1584.0 \mathrm{~s}, 1491.5 \mathrm{sh}$ \\
\hline Ring $v(N-C)$ & 1434 & $1436.6 \mathrm{~m}$ \\
\hline$\delta(\mathrm{C}-\mathrm{H})$ in plane & 1092, 1074 & 1094.0 w, 1069.7 m, 1048.9 w \\
\hline$\delta(\mathrm{C}-\mathrm{H})$ (wagging), Ring twisting & 816,730 & 753.5 vs \\
\hline Ring twisting & 646 & $643.5 \mathrm{sh}, 621.3 \mathrm{w}$ \\
\hline Ring twisting, N-H wagging & 528 & $562.2 \mathrm{~s}$ \\
\hline$v(\mathrm{Mo}=\mathrm{O})$ of cis- $\mathrm{MoO}_{2}^{2+}$ core $^{15}$ & ---- & $972.9 \mathrm{~s}, 916.4 \mathrm{~s}$ \\
\hline \multicolumn{3}{|c|}{ Table 7:(FTIR frequencies in $\left.\mathrm{cm}^{-1}\right)$} \\
\hline Mode & $\begin{array}{c}\mathrm{C}_{4} \mathrm{H}_{8} \mathrm{NCH}_{3} \\
\text { (1-Methylpyrrolidine })^{30-32}\end{array}$ & {$[8]$} \\
\hline C-H Sym. Str. & $2973 \mathrm{~s}$ & $2971.5 \mathrm{~s}$ \\
\hline C-H Asym. Str. & 2892 sh, 2833 m, 2782 s & $2727.6 \mathrm{~s}$ \\
\hline C-H Deformation & $1452 \mathrm{~s}$ & $1614.1 \mathrm{~s}, 1459.2 \mathrm{~s}$ \\
\hline C-C Str. & $1365 \mathrm{~s}$ & $1300.8 \mathrm{sh}$ \\
\hline C-N Str. & $1243 \mathrm{~s}, 1204 \mathrm{~m}, 1162 \mathrm{~s}, 1111 \mathrm{~m}$ & 1205.9 w, 1104.6 w \\
\hline C-H Bending & $1044 \mathrm{~s}$ & 1069.3 w \\
\hline $\mathrm{CH}_{2}$ Rocking & $876 \mathrm{~s}$ & $914.6 \mathrm{~s}, 851.6 \mathrm{w}, 757.6 \mathrm{~s}$ \\
\hline CNC Deformation & $577 w$ & 593.3 w \\
\hline$v(\mathrm{Mo}=\mathrm{O})$ of cis $-\mathrm{MoO}_{2}^{2+}$ core ${ }^{15}$ & --- & 983.7 vs, $914.6 \mathrm{~s}$ \\
\hline
\end{tabular}


1-Methylpyrrolidine $\mathrm{e}^{30-32}$ has $\mathrm{C}-\mathrm{H}$ symmetric stretching at $2973 \mathrm{~cm}^{-1}$ and $\mathrm{C}-\mathrm{H}$ asymmetric stretching at $2892 \mathrm{~cm}^{-1}, 2833 \mathrm{~cm}^{-1}, 2782 \mathrm{~cm}^{-1}$. [8] has $\mathrm{C}-\mathrm{H}$ symmetric stretching at $2971.5 \mathrm{~cm}^{-1}$ and $\mathrm{C}-\mathrm{H}$ asymmetric stretching at $2727.6 \mathrm{~cm}^{-1}$. Two bands at are attributable to the presence of Stretching due to cis- $\mathrm{MoO}_{2}{ }^{2+}$ core ${ }^{15}$ are observed at $983.7 \mathrm{~cm}^{-1}$ and $914.6 \mathrm{~cm}^{-1}$ in [8] (Table 7).

\section{${ }^{1} \mathrm{H}$ NMR Spectra}

1-Methylimidazole, ${ }^{14,33-35}$ in solvent $\mathrm{CDCl}_{3}$ has peaks pertaining to $\mathrm{CH}_{3}$ protons at $3.64 \mathrm{ppm}$. $\mathrm{C}_{2}-\mathrm{H}, \mathrm{C}_{4}-\mathrm{H}$ and $\mathrm{C}_{5}-\mathrm{H}$ have absorptions at $7.38 \mathrm{ppm}$, $7.01 \mathrm{ppm}$ and $6.86 \mathrm{ppm}$, respectively. NMR of [1] in solvent DMSO- $d_{6}$ reveals that peaks due to all the protons of 1-methylimidazole have shifted downfield due to decrease in electronic density of imidazole ring. Effect is inversely proportional to distance (Table 8).

1,4-Diaminobutane ${ }^{36,37}$ in solvent $\mathrm{H}_{2} \mathrm{O}$ has peaks pertaining to $\mathrm{N}-\mathrm{H}$ protons at $1.15 \mathrm{ppm}$. NMR of [2] in solvent DMSO- $d_{6}$ indicates that peaks due to $\mathrm{NH}^{2}$ protons and middle $\mathrm{CH}_{2}$ protons of 1,4-diaminobutane have shifted downfield, but peaks due to side $\mathrm{CH}_{2}$ protons have shifted up field (Table 9) due to $\mathrm{N} \rightarrow$ Mo lone pair donation.

2-Methylpyridine 21,22,38-40 in solvent $\mathrm{CDCl}_{3}$ has peaks pertaining to $\mathrm{CH}_{3}, \mathrm{C}_{1}-\mathrm{H}, \mathrm{C}_{2}-\mathrm{H}, \mathrm{C}_{3}-\mathrm{H} \& \mathrm{C}_{4}-\mathrm{H}$ at $2.54,7.12,7.53 \& 7.08$ ppm, respectively. NMR of [3] in solvent DMSO- $d_{6}$ reveals that all of these protons have deshielded due to decrease in electron density on $\mathrm{N} \rightarrow$ Mo coordination. There is not much chance of Mo-N $\pi$-bonding due to increase of electron density by methyl group on nitrogen (Table 10).

Oncomparison of NMR of 4-methylpiperidine ${ }^{41}$ in solvent $\mathrm{CDCl}_{3}$ with that of [4], [5] and [6] (Table 11 ), it is found that all peaks in these compounds except that of $\mathrm{CH}_{3}$ have shifted downfield.

Imidazole ${ }^{35,42,43}$ in solvent $\mathrm{CDCl}_{3}$ absorbs at $11.62 \mathrm{ppm}$ due to $\mathrm{N}-\mathrm{H}$ proton. It absorbs at $7.73 \mathrm{ppm}$ due to $\mathrm{C}-\mathrm{H}$ proton (between two nitrogen atoms) \& at 7.15 ppm due to $\mathrm{C}-\mathrm{H}$ protons on other two carbons. NMR of [7] in solvent DMSO- $d_{6}$ shows that protons have been deshielded due to decrease in electron density on $\mathrm{N} \rightarrow$ Mo coordination (Table 12). Due to tautomerization equilibrium two equivalent $\mathrm{C}-\mathrm{H}$ protons of imidazole are seen as singlets. $\mathrm{N}-\mathrm{H}$ proton shows downfield peak.

On comparison of NMR of 1-methylpyrrolidine $30,44,45$ with that of [8], it is seen that all absorptions show downfield trend due to decrease in electron density on $\mathrm{N} \rightarrow$ Mo coordination (Table 13).

Table 8: ('H NMR Chemical Shift in ppm)

\begin{tabular}{|c|c|c|}
\hline Protons & $\begin{array}{c}\mathrm{C}_{3} \mathrm{H}_{3} \mathrm{~N}_{2} \mathrm{CH}_{3}(1-\text { Methylimidazole })^{14,33-35} \\
\text { in solvent } \mathrm{CDCl}_{3}\end{array}$ & [1] \\
\hline $\mathrm{N}-\mathrm{CH}_{3}$ & $3.643 \mathrm{H}$ & 3.86 \\
\hline $\mathrm{C}_{2}-\mathrm{H}$ & $7.381 \mathrm{H}$ & $9.071 \mathrm{H}$ \\
\hline $\mathrm{C}_{4}-\mathrm{H}$ & $7.011 \mathrm{H}$ & $7.911 \mathrm{H}$ \\
\hline $\mathrm{C}_{5}-\mathrm{H}$ & $6.861 \mathrm{H}$ & $7.631 \mathrm{H}$ \\
\hline \multicolumn{3}{|c|}{ Table 9: ( ${ }^{1} \mathrm{H}$ NMR Chemical Shift in ppm) } \\
\hline Protons & $\begin{array}{c}\mathrm{H}_{2} \mathrm{NC}_{4} \mathrm{H}_{8} \mathrm{NH}_{2}(1,4 \text {-Diaminobutane })^{36,37} \\
\text { in solvent } \mathrm{H}_{2} \mathrm{O}\end{array}$ & {$[2]$} \\
\hline \multirow{3}{*}{$\begin{array}{c}\mathrm{NH}_{2} \\
\text { Middle } \mathrm{CH}_{2} \\
\text { Side } \mathrm{CH}_{2}\end{array}$} & $1.154 \mathrm{H}$ & $7.874 \mathrm{H}$ \\
\hline & $1.74-1.774 \mathrm{H}$ & $1.994 \mathrm{H}$ \\
\hline & $3.03-3.064 \mathrm{H}$ & $2.39-2.414 \mathrm{H}$ \\
\hline
\end{tabular}

Table 10: (1H NMR Chemical Shift in ppm)

\begin{tabular}{lcc}
\hline Protons & $\mathrm{C}_{5} \mathrm{H}_{4} \mathrm{NCH}_{3}$ (2-Methylpyridine) \\
in solvent CDCl & \\
& $2.543 \mathrm{H} \mathrm{s}$ & {$[3]$} \\
$\mathrm{CH}_{3}$ & $7.121 \mathrm{H} \mathrm{d}$ & $2.793 \mathrm{H}$ \\
$\mathrm{C}_{2}-\mathrm{H}$ & $7.531 \mathrm{H} \mathrm{t}$ & $8.071 \mathrm{H}$ \\
$\mathrm{C}_{3}-\mathrm{H}$ & $7.081 \mathrm{H} \mathrm{t}$ & $8.431 \mathrm{H}$ \\
$\mathrm{C}_{4}-\mathrm{H}$ & $8.471 \mathrm{H} \mathrm{d}$ & $7.871 \mathrm{H}$ \\
$\mathrm{C}_{5}-\mathrm{H}$ & & $8.701 \mathrm{H}$ \\
\hline
\end{tabular}

Table 11: ( ${ }^{1} \mathrm{H}$ NMR Chemical Shift in ppm)

\begin{tabular}{ccccc}
\hline Protons & $\begin{array}{c}\mathrm{C}_{5} \mathrm{H}_{9} \mathrm{NHCH}_{3} \\
\text { (4-Methylpiperidine) } \\
\text { in solvent } \mathrm{CDCl}_{3}\end{array}$ & {$[4]$} & {$[5]$} & {$[6]$} \\
\hline $\mathrm{N}-\mathrm{H}$ & $1.841 \mathrm{H}$ & $8.78-9.021 \mathrm{H}$ & $8.96-9.201 \mathrm{H}$ & $8.89-9.131 \mathrm{H}$ \\
$\mathrm{C}_{2}-\mathrm{He} \& \mathrm{C}_{6}-\mathrm{He}$ & $3.032 \mathrm{H}$ & $4.152 \mathrm{H}$ & $3.462 \mathrm{H}$ & $3.702 \mathrm{H}$ \\
$\mathrm{C}_{2}-\mathrm{Ha} \& \mathrm{C}_{6}-\mathrm{Ha}$ & $2.572 \mathrm{H}$ & $3.152 \mathrm{H}$ & $3.14-3.172 \mathrm{H}$ & $3.162 \mathrm{H}$ \\
$\mathrm{C}_{3}-\mathrm{He} \& \mathrm{C}_{5}-\mathrm{He}$ & $1.612 \mathrm{H}$ & $2.772 \mathrm{H}$ & $2.74-2.822 \mathrm{H}$ & $2.782 \mathrm{H}$ \\
$\mathrm{C}_{3}-\mathrm{Ha} \& \mathrm{C}_{5}-\mathrm{Ha}$ & $1.082 \mathrm{H}$ & $1.322 \mathrm{H}$ & $1.56-1.712 \mathrm{H}$ & $1.322 \mathrm{H}$ \\
$\mathrm{C}_{4}-\mathrm{Ha}$ & $1.451 \mathrm{H}$ & $2.031 \mathrm{H}$ & $2.50-2.511 \mathrm{H}$ & $2.511 \mathrm{H}$ \\
$\mathrm{CH}_{3}$ & $0.913 \mathrm{H}$ & $0.883 \mathrm{H}$ & $0.893 \mathrm{H}$ & $0.893 \mathrm{H}$ \\
\hline
\end{tabular}


Table 12: ( ${ }^{1} \mathrm{H}$ NMR Chemical Shift in ppm)

\begin{tabular}{ccc}
\hline Protons & $\mathrm{C}_{3} \mathrm{H}_{4} \mathrm{~N}_{2}$ (Imidazole) $)^{35,42,43}$ & {$[7]$} \\
\hline $\mathrm{N}-\mathrm{H}$ & $12.41 \mathrm{H}$ & $14.931 \mathrm{H}$ \\
C-H between two & $7.701 \mathrm{H}$ & $9.151 \mathrm{H}$ \\
nitrogen atoms & & \\
C-H on other carbons & $7.032 \mathrm{H}$ & $7.672 \mathrm{H}$ \\
\hline
\end{tabular}

Table 13: ('H NMR Chemical Shift in ppm)

\begin{tabular}{ccc}
\hline Protons & $\mathrm{C}_{4} \mathrm{H}_{8} \mathrm{NCH}_{3}$ (1-Methylpyrrolidine) $)^{30,44,45}$ & {$[8]$} \\
\hline $\mathrm{CH}_{3}$ & $2.33 \mathrm{H}$ & $3.433 \mathrm{H}$ \\
$\mathrm{C}_{2}-\mathrm{H} \& \mathrm{C} 5-\mathrm{H}$ & $2.54 \mathrm{H}$ & $2.51-2.894 \mathrm{H}$ \\
$\mathrm{C}_{3}-\mathrm{H} \& \mathrm{C} 4-\mathrm{H}$ & $1.64 \mathrm{H}$ & $1.86-1.974 \mathrm{H}$ \\
\hline
\end{tabular}

\section{Mass Spectra (LC-MS) ${ }^{46}$}

Formulae have been derived from fragmentation obtained as under.

\section{Table 14}

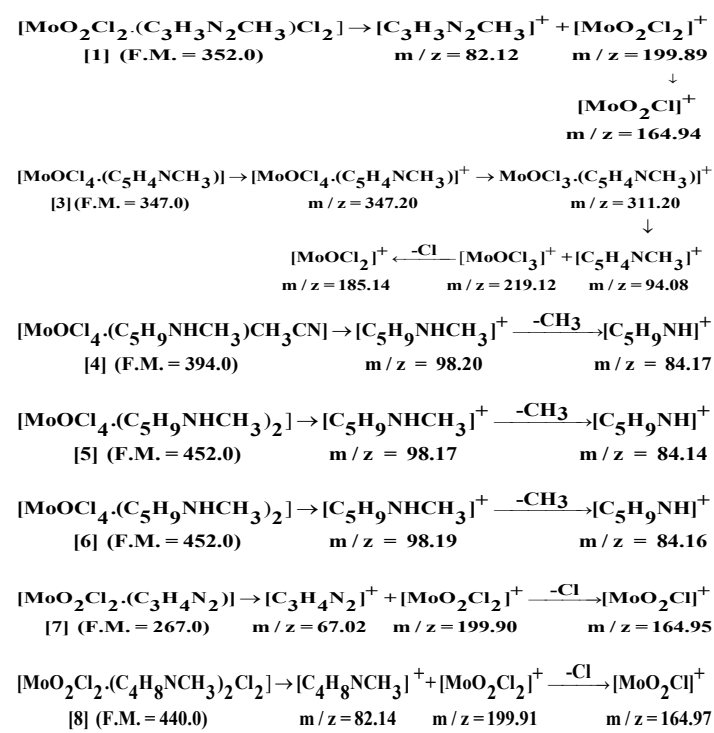

Table 15: (Fragments $\mathrm{m} / \mathrm{z}$ )

\begin{tabular}{|c|c|c|c|c|}
\hline Comp. & Fragment & Theoretical $^{46}$ & Obtained & Relative area \\
\hline \multirow[t]{3}{*}[1]{} & {$\left[\mathrm{MoO}_{2} \mathrm{Cl}_{2}\right]^{+}$} & 199.83 & 199.89 & $36 \%$ \\
\hline & {$\left[\mathrm{MoO}_{2} \mathrm{Cl}\right]^{+}$} & 164.86 & 164.94 & $20 \%$ \\
\hline & $\left.\left[\mathrm{C}_{3} \mathrm{H}_{3} \mathrm{~N}_{2} \mathrm{CH}_{3}\right)\right]^{+}$ & 82.05 & 82.12 & $95 \%$ \\
\hline \multirow[t]{5}{*}{ [3] } & {$\left[\mathrm{MoOCl}_{4}\left(\mathrm{C}_{5} \mathrm{H}_{4} \mathrm{NCH}_{3}\right)\right]^{+}$} & 346.83 & 347.20 & $5 \%$ \\
\hline & {$\left[\mathrm{MoOCl}_{3}\left(\mathrm{C}_{5} \mathrm{H}_{4} \mathrm{NCH}_{3}\right)\right]^{+}$} & 311.86 & 311.20 & $18 \%$ \\
\hline & {$\left[\mathrm{MoOCl}_{3}\right]^{+}$} & 218.80 & 219.12 & $62 \%$ \\
\hline & {$\left[\mathrm{MoOCl}_{2}\right]^{+}$} & 183.83 & 185.14 & $84 \%$ \\
\hline & {$\left[\mathrm{C}_{5} \mathrm{H}_{4} \mathrm{NCH}_{3}\right]^{+}$} & 93.05 & 94.08 & $100 \%$ \\
\hline \multirow[t]{2}{*}[4]{} & $\left.\left[\mathrm{C}_{5} \mathrm{H}_{9} \mathrm{NHCH}_{3}\right)\right]^{+}$ & 99.10 & 98.20 & $30 \%$ \\
\hline & $\left.\left[\mathrm{C}_{5} \mathrm{H}_{9} \mathrm{NH}\right)\right]^{+}$ & 84.08 & 84.17 & $10 \%$ \\
\hline \multirow[t]{2}{*}[5]{} & $\left.\left[\mathrm{C}_{5} \mathrm{H}_{9} \mathrm{NHCH}_{3}\right)\right]^{+}$ & 99.10 & 98.17 & $58 \%$ \\
\hline & $\left.\left[\mathrm{C}_{5} \mathrm{H}_{9} \mathrm{NH}\right)\right]^{+}$ & 84.08 & 84.14 & $14 \%$ \\
\hline \multirow[t]{2}{*}[6]{} & $\left.\left[\mathrm{C}_{5} \mathrm{H}_{9} \mathrm{NHCH}_{3}\right)\right]^{+}$ & 99.10 & 98.19 & $15 \%$ \\
\hline & $\left.\left[\mathrm{C}_{5} \mathrm{H}_{9} \mathrm{NH}\right)\right]^{+}$ & 84.08 & 84.16 & $6 \%$ \\
\hline \multirow[t]{3}{*}{ [7] } & {$\left[\mathrm{C}_{3} \mathrm{H}_{4} \mathrm{~N}_{2}\right]^{+}$} & 68.03 & 67.02 & $3 \%$ \\
\hline & {$\left[\mathrm{MoO}_{2} \mathrm{Cl}_{2}\right]^{+}$} & 199.83 & 199.90 & $8 \%$ \\
\hline & {$\left[\mathrm{MoO}_{2} \mathrm{Cl}\right]^{+}$} & 164.86 & 164.95 & $5 \%$ \\
\hline \multirow[t]{3}{*}[8]{} & $\left.\left[\mathrm{C}_{4} \mathrm{H}_{8} \mathrm{NCH}_{3}\right)_{2}\right]^{+}$ & 85.08 & 82.14 & $100 \%$ \\
\hline & {$\left[\mathrm{MoO}_{2} \mathrm{Cl}_{2}\right]^{+}$} & 199.83 & 199.91 & $9 \%$ \\
\hline & {$\left[\mathrm{MoO}_{2} \mathrm{Cl}\right]^{+}$} & 164.86 & 164.97 & $7 \%$ \\
\hline
\end{tabular}

\section{CONCLUSION}

In all the compounds, except [7], molybdenum to chlorine ration remains $1: 4$, which shows that polar solvent $\mathrm{CH}_{3} \mathrm{CN}$ could not solvolyze Mo-Cl bonds, thus leading to formation of adducts/ molecular complexes ${ }^{47}$.
[7] is obtained when trimethylsilylimidazole displaces chlorine from $\mathrm{MoOCl}_{4}$ to form trimethylsilylchloride and radical $\mathrm{C}_{3} \mathrm{H}_{3} \mathrm{~N}_{2}$. This radical abstracts ${ }^{2}$ hydrogen atom from the solvent $\mathrm{CH}_{3} \mathrm{CN}$ to form $\mathrm{C}_{3} \mathrm{H}_{4} \mathrm{~N}_{2}$ (imidazole).

$v(\mathrm{Mo}=\mathrm{O})$ is reported ${ }^{48}$ at $990 \mathrm{~cm}^{-1}-1010$ 
$\mathrm{cm}^{-1}$ in various inert solvents. There is a decrease in $v(\mathrm{Mo}=0)$ in [2]-[6], which shows coordination ${ }^{49}$ of ligand in a direction trans to $\mathrm{Mo}=\mathrm{O}$ bond in these adducts/molecular complexes.

There is increase in $v(\mathrm{C}=\mathrm{C})$ and $v(\mathrm{~N}-\mathrm{C})$ in [1] and [7] due to coordination of 1-methylimidazole/ imidazole to Mo through $\mathrm{N}$ atom.

Lone pair of $\mathrm{N}$ atom in [3] is involved in $\mathrm{N} \rightarrow \mathrm{Mo}$ $\mathrm{n} \pi$ conjugation as a result there is a shift of $\mathrm{v}(\mathrm{C}=\mathrm{N})$ by $29 \mathrm{~cm}^{-1}$ on higher frequency side, indicating thereby coordination of ligand ${ }^{47}$ to $\mathrm{Mo}$ through $\mathrm{N}$ atom.

Coordination in [1] and [7] takes place through N-3 of imidazole ring ${ }^{50}$.

${ }^{1} \mathrm{H}$ NMR of all compounds show downward shifts on coordination of ligands with Mo through $\mathrm{N}$ atom due to decrease in electron density of the rings.
LC-MS spectra of all the compounds synthesized prove the presence of ligands and some of the fragments in them.

\section{ACKNOWLEDGEMENT}

We acknowledge our thanks to Department of SAIF/CIL, Panjab University, Chandigarh (India) for extending us the facility for elemental analysis, FTIR, LC-MS and ${ }^{1} \mathrm{H}$-NMR to characterize compounds. Our thanks are also to Campus Director, Giani Zail Singh Campus College of Engineering \& Technology, Bathinda, Punjab (India), for providing us financial support and infrastructural facilities for this research project.

\section{Conflict of interest}

We, the authors declare that we have no conflict of interest.

\section{REFERENCES}

1. Vasisht, S. K.; Singh, G., VII International Symposium on Organosilicon Chemistry., Kyoto, Japan., 1984, 40.

2. Vasisht, S. K.; Singh, G.; Chaudhary, S., Indian Journal of Chemistry., 1985, 24A, 574-577.

3. Vasisht, S. K.; Singh, G., Z. Anorg. Allg. Chemie., 1985, 526, 161-167.

4. Vasisht, S.K.;Singh, G.;Verma, P.K. Monatshefte fur Chemie., 1986, 117, 177-183.

5. Singh, G.; Mangla, V.; Goyal, M.; Singla, K.; Rani, D., American International Journal of Research in Science, Technology, Engineering \& Mathematics., 2014, 8(2), 131-136.

6. Singh, G.; Mangla, V.; Goyal, M.; Singla, K.; Rani, D., American International Journal of Research in Science, Technology, Engineering \& Mathematics., 2015, 9(1), 25-33.

7. Singh, G.; Mangla, V.; Goyal, M.; Singla, K.; Rani, D., International Congress on Chemical, Biological and Environmental Sciences., 2015, 930-942, 7-9, Kyoto (Japan).

8. $\quad$ Singh, G.; Mangla, V.; Goyal, M.; Singla, K.; Rani, D., American International Journal of Research in Science, Technology, Engineering \& Mathematics., 2015, 10(4), 299-308.

9. $\quad$ Singh, G.; Mangla, V.; Goyal, M.; Singla, K.; Rani,
D.; Kumar, R., American International Journal of Research in Science, Technology, Engineering \& Mathematics., 2016, 16(1), 56-64.

10. Singh, G.; Kumar, R., American International Journal of Research in Science, Technology, Engineering \& Mathematics., 2018, 22(1), 01-08.

11. Mangla,V.;Singh, G., AmericanInternationalJournal of Research in Science, Technology, Engineering \& Mathematics., 2019, 26(1), 145-148.

12. Vogel, A. I., A Text Book of Quantitative Inorganic Analysis; John Wiley and Sons, New York., (Standard method)., 1963.

13. https://webbook.nist.gov/cgi/cbook.cgi?ID $=C$ 616477\&Units $=$ SI\&Mask $=80$.

14. Van K. C. G.; Reedijk, J., Inorganica Chimica Acta.,1978, 30, 171-177.

15. Abramenko, V.L.; Sergienko, V.S.; Churakov, A.V., Russian J. Coord. Chem., 2000, 26(12), 866-871.

16. Ward, B. G.; Stafford, F. E., Inorg. Chem., 1968, 7, 2569.

17. Bodo, H. H.; Regina, Z. Chem., 1976, 16, 407.

18. Ergu "N. Kasap; Su" Leyman; O" Zceli'K., J. Inclusion Phenomena and Molecular Recognition in Chem., 1997, 28, 259-267.

19. http://www.hanhonggroup.com/ir/ir_en/ B61062.html. 
20. Arici K; Gul, O., International J. Chemistry and Technology., 2018, 2(2), 141-152,

21. Hossain, A. G. M. M.; Ogura, K., Indian J. Chem., 1996, 35A, 373-378.

22. Gupta, S. K.; Srivastava, T. S., J. Inorganic and Nuclear Chem., 1970, 32, 1611-1615.

23. https://www.sigmaaldrich.com/spectra/ftir/ FTIR008407.PDF.

24. Gulluoglu, M. T.; Erdogdu, Y.; Yurdakul, S., J. Molecular Structure., 2007, 834-836. 540-547.

25. Fabretti, A. C.; Franchini G. C.; Preti, C.; Tosi, G.; Zannini, P., Transition Metal Chem., 1985, 10, 284-287.

26. Manhas, B.S.; Pal, S.; Trikha A. K., Indian J. Chem., 1991, 30A, 638-640.

27. Naji A. A.; AL-Askari, M.; Saed, B. A., Basrah Journal of Science (C), 2012, 30, 119-131.

28. Mohan, J., Organic Spectroscopy: Principles and Applications, CRC Press., 2004.

29. Hodgson, J.B.; Percy, G. C.; Thornton, D.A., J. Molecular Structure., 1980, 66, 81-92.

30. Hoa, N. V.; Tuan, N. A.; Thao, P.T.; Huyen, T.T. T., Journal of Science and Technology., 2016, 54(2), 231-237.

31. https://www.sigmaaldrich.com/spectra/ftir/ FTIR008415.PDF.

32. Szafran, M.; Koput, J.; Szafran, Z. D.; Kwiatkowski, J.S., Vibrational Spectroscopy., 2000, 23, 1-11.

33. https://www.chemicalbook.com/Spectrum EN_616-47-7_1HNMR.htm.

34. http://www.hanhonggroup.com/nmr/nmr_en/ B42171.html.
35. Zamani, K.; Khaledi, M.; Foroughifar, N.; Mahdavi, V., Turk. J. Chem., 2003, 27, 71-75

36. http://www.ymdb.ca/compounds/YMDB00 132.

37. http://www.hmdb.ca/spectra/spectra/nmr_ one_d/1703.

38. http://www.hanhonggroup.com/nmr/nmr_en/ B61062.html.

39. http://www.sigmaaldrich.com/spectra/fnmr/ FNMR000256.PDF.

40. Kumari, N.; Sharma, M.; Das,, P.; Dutta, D. K., Applied Organomet. Chem., 2002, 16, 258-264.

41. https://sdbs.db.aist.go.jp/sdbs/cgi-bin/direct frame_disp.cgi?sdbsno=591\&spectrum_ type=HNMR\&fname=HSP40452.

42. Nuran Özçiçek Pekmez; Muzaffer Can; Attila Yildiza, Acta Chim. Slov., 2007, 54, 131-139.

43. http://www.hmdb.ca/spectra/nmr_one_d/ 1723.

44. https://www.sigmaaldrich.com/spectra/fnmr/ FNMR010994.PDF.

45. http://www1.chem.umn.edu/groups/taton/ chem8361/Problem\%20Sets/Workshop\%20 1\%20Solutions--2012.pdf.

46. http://www.sisweb.com/referenc/tools/ exactmass.htm.

47. Abramenko, V. L.; Russian J. Coord. Chem., 2001, 27(11), 819-822.

48. Barraclough, C. G.; Kew, D. J., Australian J. Chem., 1970, 23, 2387-2396.

49. Abramenko, V. L.; Sergienko, V. S., Russian J. Inorg. Chem., 2009, 54(13), 2031-2053.

50. Ritterskamp, N.; Sharples, K.; Richards, E.; Folli, A.; Chiesa, M.; Platts, J. A.; Murphy, D. M., Inorganic Chemistry., 2017, 56, 11862-11875. 